\title{
Reporting of vertebral fragility fractures: can radiologists help reduce the number of hip fractures?
}

\author{
R. M. Mitchell ${ }^{1}$ P. Jewell ${ }^{2}$ M. K. Javaid ${ }^{3} \cdot$ D. McKean ${ }^{4} \cdot$ S. J. Ostlere ${ }^{5}$
}

Received: 20 December 2016 / Accepted: 24 July 2017 /Published online: 7 August 2017

(C) The Author(s) 2017. This article is an open access publication

\begin{abstract}
Summary Patients with osteoporotic vertebral fractures are at increased risk of hip fracture. In a cohort of hip fracture patients, many had previous imaging studies showing incidental vertebral fractures. Fifty-four percent of fractures were not reported by the radiologist, highlighting a missed opportunity for diagnosing and treating osteoporosis, thereby preventing further fractures.

Purpose Patients with osteoporotic vertebral fragility fractures (VFFs) are at increased risk of future fractures, including hip fractures. Treating osteoporosis in these patients has the potential to reduce the risk of subsequent hip fractures, which are associated with high morbidity, mortality and cost. In this retrospective cohort study, we investigated the reporting and follow-up of VFFs evident on imaging by radiologists at the John Radcliffe Hospital, Oxford.

Materials and methods Data from the local Fracture Liaison Service was used to case-find all incident hip fractures from 2013 presenting to the trust. We then identified patients who had also undergone a radiological procedure that included the
\end{abstract}

R. M. Mitchell and P. Jewell contributed equally to this work

R. M. Mitchell

ruth.mitchell@doctors.org.uk

1 Merton College, University of Oxford, Oxford, UK

2 New College, University of Oxford, Oxford, UK

3 Nuffield Department of Orthopaedics, Rheumatology and Musculoskeletal Sciences, University of Oxford, Oxford, UK

4 Stoke Mandeville Hospital, Buckinghamshire Hospital NHS Trust, Aylesbury, UK

5 Nuffield Orthopaedic Centre, Oxford University Hospital NHS Trust, Oxford, UK thoracic and/or lumbar spine in the previous 6 years. All identified radiological images were re-examined for the presence of VFFs using the Genant semi-quantitative method.

Results Seven hundred and thirty-two patients over the age of 50 with a hip fracture in 2013 were identified. One hundred and fifty-seven patients had previously undergone a radiological procedure involving the spine, and VFFs were identified in $65 / 157(41 \%)$. Of these, only $30 / 65(46 \%)$ were reported by a radiologist when the fracture was first visible. 32/35 (91\%) of unreported VFFs were from imaging reported by nonmusculoskeletal radiologists. Only 16/65 (25\%) of patients with a VFF were documented as being on bone-specific therapy at the time of hip fracture.

Conclusions Our study highlights the under-reporting of osteoporotic vertebral fractures, particularly by nonmusculoskeletal radiologists. Better systems for reporting and referring osteoporotic VFFs are necessary to increase the number of patients receiving appropriate osteoporosis treatment.

Keywords Vertebral fragility fracture $\cdot$ Hip fracture $\cdot$

Osteoporosis $\cdot$ Bone protection

\section{Introduction}

Osteoporosis is a systemic skeletal disorder characterised by low bone mass and microarchitectural deterioration resulting in an increased bone fragility and susceptibility to fracture [1]. Sufferers of osteoporosis are at an increased risk of fracture. One in 2 women and 1 in 5 men over the age of 50 will experience an osteoporotic fragility fracture in their lifetime [2]. Vertebral fragility fractures (VFFs) are the most common osteoporotic fracture [3]. VFFs can be identified from images performed specifically for examination of the spine (e.g. 
spinal radiographs, DXA vertebral fracture assessment) or incidentally (opportunistically) from images performed for other clinical indications (e.g. CT, MRI, lateral chest radiograph, abdominal radiograph) [4]. Despite being a common incidental finding on diagnostic imaging, the majority of fractures are asymptomatic [5]. However, it is well established that individuals with VFFs are at a significantly increased risk of suffering hip fractures and further vertebral fractures [6, 7]. Hip fractures are associated with increased morbidity and mortality, and in the UK account for at least $£ 1.1$ billion in hospital costs alone [8, 9].

VFFs seen on imaging are significantly under-diagnosed by radiologists [4], meaning a large proportion of patients are not receiving suitable, effective osteoporosis treatment. Reporting VFFs is an important component of the International Osteoporosis Foundation (IOF) Best Practice Framework for secondary fracture prevention $[10,11]$. The recognised under-reporting of vertebral fractures stimulated the development of the Vertebral Fracture Initiative of the IOF [12], which provides an educational resource on the detection and management of these fractures for radiologists, clinicians and other healthcare professionals. Knowing that a patient has a vertebral fracture will also give more accurate assessments of future fracture risk using the WHO Fracture Risk Assessment Tool (FRAX) [13]. There are a wide range of treatments available for osteoporosis, which can reduce the incidence of future fractures by $30-65 \%$ [14]. Therefore, reporting of serendipitous VFFs by radiologists, and appropriate follow-up by the referring clinician, can help to reduce the number of hip fractures, and as a result, patient suffering and financial burden on the NHS.

In this retrospective cohort study, we investigated the prevalence and severity of osteoporotic vertebral fractures within a cohort of older patients who had recently suffered hip fractures. We then reviewed whether these vertebral fractures had been reported by the interpreting radiologist at the time of imaging.

\section{Methods}

\section{Patient identification}

A search was performed using our departmental radiology information database (RIS). This was used to identify all patients over the age of 50 who had suffered a hip fracture in 2013. From this cohort, we identified a subset of patients who also had imaging of the spine in the preceding 5 years within our NHS Trust, which included any CT (chest, abdomen, pelvis, colon or other), MRI (spine or other) or plain film (spine or lateral chest radiograph) in which at least five thoracic and/ or lumbar vertebral bodies were clearly visible. Patients with the following conditions stated in the imaging request details or radiologist's report were excluded from subsequent analysis: pubic rami fractures, peri-prosthetic fractures, history of high-velocity trauma, primary or metastatic bone malignancy, multiple myeloma or disorders of bone such as osteogenesis imperfect. Other exclusion criteria included unobtainable or poor quality images. Vertebral deformities were not recorded as fractured if judged to be secondary to Schmorl's nodes or degenerative disc disease (spondylosis). Assessment of spinal imaging was also based on published normative values of anterior vertebral body vertical height to posterior vertebral body vertical height ratio to identify physiological vertebral wedging, most commonly seen at the thoracolumbar junction [15]. This study was registered as audit with our institution audit department.

\section{Identification of vertebral fractures}

Imaging of the spine was assessed for the presence of vertebral fractures using the Genant semi-quantitative classification [16], classing fractures as grade 1 (20-25\% height reduction), 2 (26-40\% height reduction) or 3 (> 40\% height reduction) depending on the extent of vertebral height loss by PJ and RM (medical students). All fractures identified using this method were reviewed by a consultant musculoskeletal radiologist (SO) to verify the presence of fractures, and to exclude vertebral deformity secondary to other factors. All reports from spinal imaging were evaluated for satisfactory reporting of any VFFs. Cumulative fracture score was calculated by the sum of the Genant fracture scores in a particular radiological image. Musculoskeletal radiologists were defined as those reporting imaging for musculoskeletal indications (X-ray spine or MRI spine).

\section{Treatment data}

Treatment data was obtained from our institution's Fracture Prevention Service database.

\section{Results}

Seven hundred and thirty-two patients aged 50 years or above who had a hip fracture in 2013 were identified from our PACS database. Of these, 169 had X-ray, CT or MRI imaging of the spine on one or more occasions during the 5 years prior to their fracture. Twelve patients were excluded from subsequent analysis for the following reasons: unable to fetch/view scan (5), malignancy with bone metastases (4), osteogenesis imperfecta (1), bone lytic lesions (1) and high impact trauma (1). Of the remaining 157 patients, 66\% were female, and mean age at the time of hip fracture was 82.5 years, with a range of 56 to 100 years. 


\section{Reporting of vertebral fractures by radiologists}

Of the 157 individuals who had spinal imaging prior to their hip fracture, 65 (41\%) had detectable VFFs (Table 1). Only 30 $(46 \%)$ of the VFFs first detectable on imaging were satisfactorily reported as 'fractured' by the interpreting radiologist at the time of imaging (Table 2), an example given in Fig. 1. Seven fractures were reported on a later date after additional spinal imaging. In imaging reported by musculoskeletal radiologists, $89 \%$ of fractures were reported, whereas in imaging reported by non-musculoskeletal radiologists only $14 \%$ of fractures were reported.

In images where the most severe grade of fracture was grade 1 , only $7 \%$ of fractures were reported, compared with $68 \%$ of images where grade 2 was the most severe fracture, and $75 \%$ of images where grade 3 was the most severe (Fig. 2 ). The mean cumulative fracture score for VFFs on imaging where the fracture had been reported was 4.80, which was significantly higher than that for imaging where VFFs were not reported $($ mean $=2.91 ; p=0.012$ ). The number of vertebral fractures was also significantly higher on imaging where VFFs had been reported (mean $=2.40$ ) compared with imaging where VFFs were not reported $($ mean $=1.69 ; P=0.025)$.

\section{Bone-specific treatment in patients with vertebral fractures}

Of those patients with a previously detectable VFF, only $16 / 65$ $(25 \%)$ were documented as taking bone-specific therapy at the time of hip fracture. Of these, 9 patients were taking alendronate, 3 risedronate and 4 calcium and vitamin D (data not shown). Of the 16 receiving therapy, a vertebral fracture had been reported in 13 patients (including 4 that were not reported at the first opportunity, but reported following later imaging). It was not possible to tell from our database whether treatment had been started because of a vertebral fracture being identified.

\section{Discussion}

Our results reveal several important findings. Firstly, of the cohort of hip fracture patients, a significant proportion (over a fifth) had undergone previous imaging that included the spine. Of these patients, $41 \%$ had a detectable VFF. Taken together, these findings indicate a potential opportunity for detecting incidental vertebral fractures at an early stage.

Secondly, our data demonstrate that VFFs are significantly underreported by radiologists, particularly by nonmusculoskeletal radiologists. Only $46 \%$ of visible vertebral fractures were recorded in the written report by the reporting radiologist at the time. However, the majority of fractures were recorded in reports by musculoskeletal radiologists. Non-musculoskeletal radiologists were less likely to identify vertebral fractures, particularly those of a lower Genant grade. On spinal X-rays, where the primary aim of imaging is to identify bony deformities, all fractures were reported. This suggests that it is not the detection of VFFs that presents a challenge to the radiologist, but lack of awareness of the need to look specifically for them. Vigilance for vertebral fractures should be increased in a population such as this where mean age is over 80 and therefore a high incidence of vertebral fractures should be anticipated [17].

Under-reporting of vertebral fractures by radiologists has been identified previously in several studies. Gehlbach et al. investigated the reporting of VFFs on lateral chest radiographs and found that only $52 \%$ of radiology reports contained notation of the presence of a vertebral fracture in the narrative of the report [18]. Vertebral fractures have also been shown to be under-reported on CT scans of the abdomen and/or pelvis [19] and MRI scans of the breast [20].

We also show that VFFs are more likely to be reported if there are multiple fractures, or if the fracture is more severe. This suggests that fractures causing obvious deformity to the spine are more likely to be commented on. However, in many cases, the spine may not be studied in sufficient detail to identify milder, less obvious fractures.

Only $25 \%$ of patients with vertebral fractures were reported to be receiving bone protection treatment at the time of their hip fracture. There are several reasons why patients with vertebral fractures may not go on to receive treatment for osteoporosis. Firstly, as we have demonstrated, vertebral fractures are often not reported by the radiologist, and at-risk patients are therefore not identified. Secondly, there may not be a robust system in place to ensure that patients who are discovered to have an osteoporotic fracture are referred for further followup and treatment. This is supported by a recent review of fracture liaison services globally which demonstrated that few hospitals have such a system of linking serendipitous vertebral fracture case finding for fracture liaison services
Table 1 Demographic of study cohort, with and without detectable VFFs

\begin{tabular}{lll}
\hline & No VFF detectable on imaging $(n=92)$ & VFF detectable on imaging $(n=65)$ \\
\hline Hip fracture $(n=157)$ & $92(59 \%)$ & $65(41 \%)$ \\
Age (mean in years, range) & $82(56-94)$ & $83(62-100)$ \\
Gender $(\%$ female $)$ & $69 \%$ & $63 \%$ \\
\hline
\end{tabular}

$V F F$ vertebral fragility fracture 
Table 2 Comparison of VFF reporting in hip fracture patients with VFF detectable on imaging

\begin{tabular}{|c|c|c|}
\hline & \multicolumn{2}{|c|}{ Number of patients with detectable VFF on imaging $(n=65)$} \\
\hline & $\begin{array}{l}\text { VFF reported at first } \\
\text { opportunity }(n=30)\end{array}$ & $\begin{array}{l}\text { VFF not reported at first } \\
\text { opportunity }(n=35)\end{array}$ \\
\hline $\begin{array}{l}\text { Number of patients with detectable VFF on } \\
\text { imaging }(n=65)\end{array}$ & $30(46 \%)$ & $35(54 \%)$ \\
\hline Age (mean in years; range) & $84(68-100)$ & $83(62-100)$ \\
\hline Gender ( $\%$ female $)$ & $67 \%$ & $60 \%$ \\
\hline \multicolumn{3}{|l|}{ VFF details } \\
\hline Cumulative fracture score (mean; SD) & $4.5(3.20)$ & $2.9(2.23)$ \\
\hline No. of fractures (mean; SD) & $2.4(1.65)$ & $1.7(1.05)$ \\
\hline \multicolumn{3}{|l|}{ Imaging details $(n, \%)$} \\
\hline MRI spine ${ }^{\mathrm{a}}$ & $7(23 \%)$ & $3(9 \%)$ \\
\hline MRI other & $0(0 \%)$ & $0(0 \%)$ \\
\hline CT chest, abdomen, pelvis & $2(7 \%)$ & $3(9 \%)$ \\
\hline $\mathrm{CT}$ chest \pm abdomen $^{\mathrm{b}}$ & $2(7 \%)$ & $7(20 \%)$ \\
\hline $\mathrm{CT}$ abdomen \pm pelvis & $0(0 \%)$ & $3(9 \%)$ \\
\hline CT colon & $1(3 \%)$ & $7(20 \%)$ \\
\hline CT other & $0(0 \%)$ & $8(23 \%)$ \\
\hline X-ray spine ${ }^{a}$ & $18(60 \%)$ & $0(0 \%)$ \\
\hline Lateral chest X-ray & $0(0 \%)$ & $4(11 \%)$ \\
\hline
\end{tabular}

$V F F$ vertebral fragility fracture, $S D$ standard deviation

${ }^{\text {a }}$ Thoracic and/or lumbar spine

${ }^{\mathrm{b}}$ Including CT pulmonary angiogram
[21]. Currently in our Trust, it is the responsibility of the general practitioner to follow up on the fracture and initiate further investigations and treatment. If the imaging was carried out for a complaint unrelated to the spine, treating the incidental finding of a vertebral fracture may not be prioritised

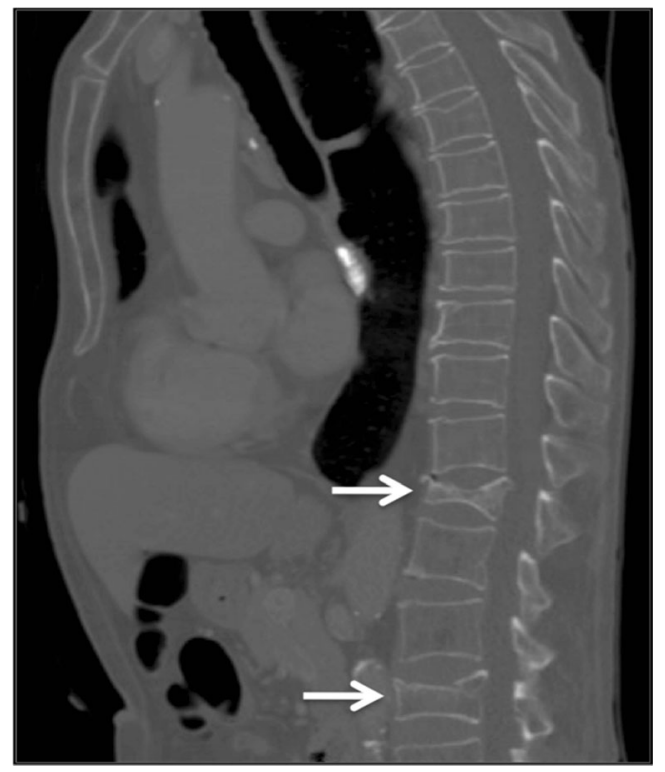

Fig. 1 A sagittal section from a CT scan of a patient aged 71 years. The grade 3 L1 fracture and grade 2 L4 fracture (indicated) were not reported by the radiologist. The patient went on to suffer a hip fracture 3 years later or pursued. The county's fracture liaison service is therefore currently unaware of a significant number of treatable cases of osteoporosis. To address this, we intend to introduce a system whereby patients with a vertebral fracture identified on a radiological report are directly referred to the local Fracture Prevention Service. A specialist fracture prevention nurse will automatically receive a list of all the vertebral fracture patients identified each month and will arrange follow-up investigations or treatment. Patients with a newly diagnosed VFF can

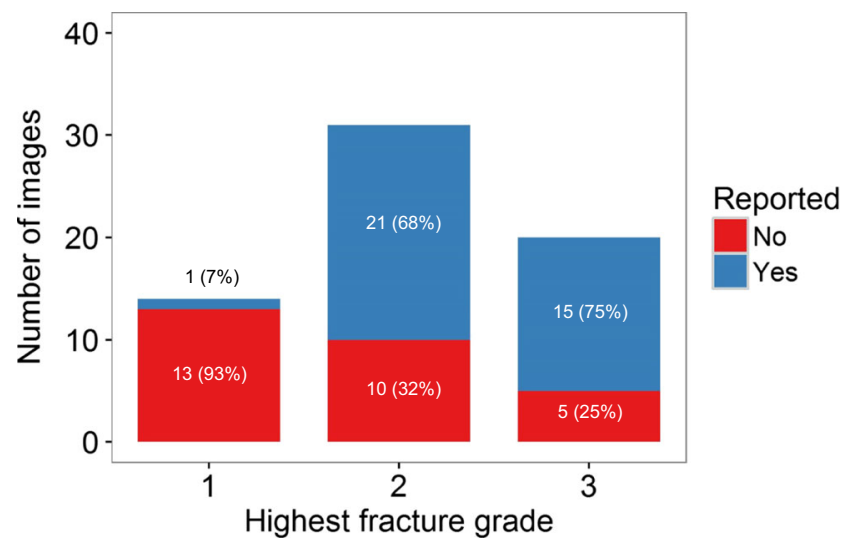

Fig. 2 Number of images where the most severe grade of fracture was 1, 2 or 3 . The blue part of each bar represents the number of images where a VFF was reported, and the red part represents images where the fracture was not reported 
then be easily identified from radiological reports and referred for appropriate follow-up and management.

Our study has some limitations. The retrospective nature of the study introduces an observer bias. We did not collect data on patients' primary diagnosis or the indication for their imaging. We therefore cannot comment as to whether in studies with significant intra-thoracic or intra-abdominal findings, VFF were more likely to be overlooked. It is not always straight-forward differentiating fracture from deformity and the following factors are considered by the radiologist when making the distinction: presence of endplate abnormalities, loss of vertebral disc height and appearance in comparison to adjacent vertebrae. Even with a standardised method such as the Genant semi-quantitative technique, there is a room for subjectivity in the interpretation of imaging, particularly for grade 1 fractures. Finally, we had limited data on patients' contraindications to osteoporosis medication. We therefore cannot comment on the number of cases where bone protection could not be commenced as a result of contraindications to therapy such as drug hypersensitivity, mechanical problems of the oesophagus (such as oesophageal stricture, dysmotility or achalasia) or those with severe renal dysfunction. Finally, we could not identify cross sectional imaging performed by hospitals outside the catchment of our NHS Trust.

\section{Conclusions}

A significant proportion of patients with osteoporotic hip fractures have had previous spinal imaging in which VFFs are detectable. VFFs are more likely to be reported if there are multiple fractures, if the fracture is more severe or if reported by a musculoskeletal radiologist. However, less than half of detectable VFFs are reported at the time of imaging by the interpreting radiologist, representing consistent underdiagnosis of these fractures. This is a missed opportunity for the identification of patients at risk of further fractures. It is essential that radiologists have a high degree of vigilance for the presence of VFFs on routine imaging, particularly in older patients, to allow appropriate follow-up and initiation of effective osteoporosis treatment. This may have a significant impact in reducing the number of subsequent hip fractures and the associated morbidity, mortality and financial burden.

\section{Compliance with ethical standards}

Funding No funding was received for this study.

\section{Conflicts of interest None.}

Ethical approval All procedures performed in studies involving human participants were in accordance with the ethical standards of the institutional and/or national research committee and with the 1964
Helsinki declaration and its later amendments or comparable ethical standards.

Informed consent All data used in this paper was anonymised. This article did not contain any identifiable patient data.

Open Access This article is distributed under the terms of the Creative Commons Attribution 4.0 International License (http:// creativecommons.org/licenses/by/4.0/), which permits unrestricted use, distribution, and reproduction in any medium, provided you give appropriate credit to the original author(s) and the source, provide a link to the Creative Commons license, and indicate if changes were made.

\section{References}

1. Roy D (2012) Osteoporosis: advances in assessment and drug therapy. Arthritis Research UK Topical Reviews 11 http://www. arthritisresearchuk.org/health-professionals-and-students/reports/ topical-reviews/topical-reviews-spring-2012.aspx. Accessed 20 December 2016

2. Kanis JA, Glüer CC (2000) An update on the diagnosis and assessment of osteoporosis with densitometry. Committee of Scientific Advisors, International Osteoporosis Foundation. Osteoporos Int 11:192-202. doi:10.1007/s001980050281

3. Cummings SR, Melton LJ (2002) Epidemiology and outcomes of osteoporotic fractures. Lancet 359:1761-1767. doi:10.1016/S01406736(02)08657-9

4. Adams JE (2016) Opportunistic identification of vertebral fractures. J Clin Densitom 19:54-62. doi:10.1016/j.jocd.2015.08.010

5. Cooper C, Atkinson EJ, O'Fallon WM, Melton LJ (1992) Incidence of clinically diagnosed vertebral fractures: a population-based study in Rochester, Minnesota, 1985-1989. J Bone Miner Res 7:221227. doi:10.1002/jbmr.5650070214

6. Black DM, Arden NK, Palermo L, Pearson J, Cummings SR (1999) Prevalent vertebral deformities predict hip fractures and new vertebral deformities but not wrist fractures. Study of Osteoporotic Fractures Research Group J Bone Miner Res 14:821-828. doi:10. 1359/jbmr.1999.14.5.821

7. Melton LJ, Atkinson EJ, Cooper C, O'Fallon WM, Riggs BL (1999) Vertebral fractures predict subsequent fractures. Osteoporos Int 10:214-221. doi:10.1007/s001980050218

8. Leal J, Gray AM, Prieto-Alhambra D et al (2016) Impact of hip fracture on hospital care costs: a population-based study. Osteoporos Int 27:549-558. doi:10.1007/s00198-015-3277-9

9. NICE (2012) Osteoporosis: assessing the risk of fragility fracture. Guidance and guidelines. https://www.nice.org.uk/guidance/ cg146. Accessed 20 November 2016

10. Åkesson K, Marsh D, Mitchell PJ et al (2013) Capture the fracture: a best practice framework and global campaign to break the fragility fracture cycle. Osteoporos Int 24:2135-2152. doi:10.1007/s00198013-2348-z

11. Marsh D, Åkesson K, Beaton DE et al (2011) Coordinator-based systems for secondary prevention in fragility fracture patients. Osteoporos Int 22:2051-2065. doi:10.1007/s00198-011-1642-x

12. International Osteoporosis Foundation (2012) Vertebral Fracture Initiative: Vertebral fracture teaching programme https://www. iofbonehealth.org/what-we-do/training-and-education/educationalslide-kits/vertebral-fracture-teaching-program. Accessed: 15 March 2016

13. Kanis JA, Borgstrom F, De Laet C et al (2005) Assessment of fracture risk. Osteoporos Int 16:581-589. doi:10.1007/s00198004-1780-5 
14. Delmas PD (2002) Treatment of postmenopausal osteoporosis. Lancet 359:2018-2026. doi:10.1016/S0140-6736(02)08827-X

15. Matsumoto M, Okada E, Kaneko Y et al (2011) Wedging of vertebral bodies at the thoracolumbar junction in asymptomatic healthy subjects on magnetic resonance imaging. Surg Radiol Anat 33:223228. doi:10.1007/s00276-010-0746-x

16. Genant HK, Wu CY, van Kuijk C, Nevitt MC (1993) Vertebral fracture assessment using a semiquantitative technique. J Bone Miner Res 8:1137-1148. doi:10.1002/jbmr.5650080915

17. Sambrook P, Cooper C (2006) Osteoporosis. Lancet 367:2010 2018. doi:10.1016/S0140-6736(06)68891-0

18. Gehlbach SH, Bigelow C, Heimisdottir M et al (2000) Recognition of vertebral fracture in a clinical setting. Osteoporos Int 11:577582. doi: $10.1007 / \mathrm{s} 001980070078$
19. Bartalena T, Giannelli G, Rinaldi MF et al (2009) Prevalence of thoracolumbar vertebral fractures on multidetector CT: underreporting by radiologists. Eur J Radiol 69:555-559. doi:10. 1016/j.ejrad.2007.11.036

20. Bazzocchi A, Spinnato P, Garzillo G et al (2012) Detection of incidental vertebral fractures in breast imaging: the potential role of MR localisers. Eur Radiol 22:2617-2623. doi:10.1007/s00330012-2521-3

21. Javaid MK, Kyer C, Mitchell PJ et al (2015) Effective secondary fracture prevention: implementation of a global benchmarking of clinical quality using the IOF Capture the Fracture ${ }^{\circ}$ Best Practice Framework tool. Osteoporos Int 26:2573-2578. doi:10.1007/ s00198-015-3192-0 